Pacific Journal of Mathematics

ON TOTALLY DIFFERENTIABLE AND SMOOTH FUNCTIONS 


\title{
ON TOTALLY DIFFERENTIABLE AND SMOOTH FUNCTIONS
}

\author{
HASSLER WHITNEY
}

1. Introduction. H. Rademacher has proved that a function of $n$ variables satisfying a Lipschitz condition is totally differentiable a. e. (almost everywhere) (see, for instance, Saks, [6, pp. 310-311]). It was discovered by H. Federer (though not stated as a theorem; see [2, p. 442]) that if $f$ is totally differentiable a.e. in the bounded set $P$, then there is a closed set $Q \subset P$ with the measure $|P-Q|$ as small as desired, such that $f$ is smooth (continuously differentiable) in $Q$; that is, the values of $f$ in $Q$ may be extended through space so that the resulting function $g$ is smooth there.

Theorem 1 of the present paper strengthens the latter theorem by showing that $f$ is approximately totally differentiable a.e. in $P$ if and only if $Q$ exists with the above property. The rest of the paper gives further theorems in the direction of Federer's Theorem, as follows.

Suppose the domain of definition of $f$ were a bounded open set $P$. Then in applying the part $(\mathrm{a}) \longrightarrow(\mathrm{c})$ of The orem 1 , we might alter $f$ in a set $P-Q$ which included a neighborhood of the boundary of $P$. In applications, it might be important to keep the values of $f$ in most of a subset close to the boundary of $P$, or in most of some other subset. That such can be done follows from Theorem 2 .

If $f$ satisfies a Lipschitz condition, Theorem 3 shows that $g$ may be made to satisfy a Lipschitz condition also, with a constant which equals a number $\rho_{n}$ (depending on the number $n$ of variables only) times the constant for $f$; in the case of one variable, we may take $\rho_{1}=1$.

If we weaken the assumption on $f$, assuming only that it is measurable, then Lusin's Theorem shows that we can alter $f$ on a set of arbitrarily small measure, giving a continuous function $g$. In the other direction, suppose we assume that $f$ (defined in an open set) has continuous $m$ th partial derivatives, and that these derivatives are totally differentiable a.e. Then Theorem 4 shows that we may alter $f$ on a set of arbitrarily small measure, giving a function $g$ which has continuous partial derivatives of order $m+1$. For the case of one variable, this is essentially a theorem of Marcinkiewicz, [5, Theorem 3].

Examples show that the hypotheses in the theorems cannot be materially 
weakened without altering the conclusions. For instance, define a function $\phi$ of one variable as follows. Let $\phi_{0}(t)$ be the distance from $t$ to the nearest integer. Using any sufficiently large integer $a$, set

$$
\phi_{i}(t)=2^{i} \phi_{0}\left(a^{i} t\right) / a^{i}, \quad \phi(t)=\sum_{i=0}^{\infty} \phi_{\imath}(t) .
$$

Then $\phi$ satisfies a Lipschitz condition of order $1-\alpha$, for any $\alpha>0$; but Property (c) of Theorem 1 is not true for it. If $\Phi(t)=\int_{0}^{t} \phi(s) d s$, then $\Phi$ is smooth, and its first derivative satisfies a Lipschitz condition of order $1-\alpha$; but the conclusion of Theorem 4 (with $m=1$ ) fails.

2. The theorem for bounded sets. Let $x=\left(x_{1}, \cdots, x_{n}\right)$ denote points of $n$-space $E^{n}$. With the unit vectors $e_{1}, \cdots, e_{n}$ of a coordinate system, any vector $v$ can be written in the form $\Sigma v_{i} e_{i}$. The length of $v$ is $|v|=\left(\sum v_{i}^{2}\right)^{1 / 2} ;|y-x|$ is the distance from $x$ to $y$. Given $n$ numbers $f_{1}(x), \cdots, f_{n}(x)$, set

$$
F(x) \cdot v=\sum f_{k}(x) v_{k}
$$

this is linear in $v$. If $f(x), f(y)$, and the $f_{k}(x)$ are defined, set

$$
e(x, y)=\frac{f(y)-f(x)-F(x) \cdot(y-x)}{|y-x|}
$$

for $y \neq x$, and $e(x, x)=0$. Let $S_{z}[\alpha(z)]$ denote the set of elements $z$ with the property $a$. Given $f$, and so on, as above, set

$$
H(x, \epsilon)=S_{y}[e(x, y)<\epsilon] .
$$

The measurable function $f$ defined in the set $P$ is a.t.d. (approximately totally differentiable) at $x \in P$ in terms of the $f_{k}(x)$ (see [6, p. 300]) if for each $\epsilon>0$ the set $H(x, \epsilon)$ has $x$ as a point of density. (Any standard definition of density points may be used for the purposes of this paper.) If this holds, then $x$ is a point of density of $P$, and the $f_{k}(x)$ are uniquely determined; if $x$ is a point of density in the direction of each axis, then the $f_{k}(x)$ are the approximate partial derivatives of $f$ at $x$. The $f_{k}$ are measurable (see $[6$, p. 299]).

THEOREM 1. Let $f$ be measurable in the bounded set $P$. Then the four following conditions are equivalent: 
(a) The function fis a.t.d.a.e. in P.

(b) The function $f$ is approximately derivable with respect to each variable a.e. in $P$.

(c) For each $\epsilon>0$ there is a closed set $Q \subset P$ such that $|P-Q|<\epsilon$ and $f$ is smooth in $Q$.

(d) There is a sequence of disjoint closed sets $Q_{1}, Q_{2}, \cdots$ in $P$ such that $\left|P-Q_{1} \cup Q_{2} \cup \cdots\right|=0$ and $f$ is smooth in each $Q_{i}$.

REMARK. If $f$ is assumed totally differentiable a.e. in $P$, the proof that (c) holds is simplified; see [2, p. 442].

Proof of Theorem 1. For the equivalence of (a) and (b), see [6, pp. 300-303]. Note that (b) is an obvious consequence of (d). We shall prove the equivalence of (a), (c) and (d).

Suppose (c) holds. We choose the disjoint closed sets $Q_{1}, Q_{2}, \cdots$ in succession so that $f$ is smooth in each and $\left|P_{i}\right| \leq|P| / 2^{i}$, where

$$
P_{i}=P-Q_{1} \cup \cdots \cup Q_{i},
$$

as follows. Having found $Q_{1}, \cdots, Q_{i-1}$, choose a closed set $Q_{i}^{\prime}$ so that $f$ is smooth in $Q_{i}^{\prime}$ and $\left|P-Q_{i}^{\prime}\right| \leq|P| / 2^{i+1}$. Let $U_{\delta}(A)$ denote the $\delta$-neighborhood of the set $A$. For small enough $\delta$, we may use

$$
Q_{i}=Q_{i}^{\prime}-U_{\delta}\left(Q_{1} \cup \cdots \cup Q_{i-1}\right) .
$$

Thus (d) holds.

Suppose (d) holds. Let $Q_{i}^{*}$ be the set of points of density of $Q_{i}$, and set $Q^{*}=Q_{1}^{*} \cup Q_{2}^{*} \cup \cdots$. Then $\left|P-Q^{*}\right|=0$. Take any $x \in Q^{*}$; say $x \in Q_{i}^{*}$. Since $f$ is smooth in $Q_{i}$ and $x$ is a point of density of $Q_{i}, f$ (considered now in $P$ ) is a.t.d. at $x$. Thus $f$ is a.t. d. at all points of $Q^{*}$, and (a) holds.

Now given (a), we must prove (c). There is a number $a>0$ with the following property. For any points $x, y$, and number $r$ with $|y-x| \leq r$, we have

$$
\left|U_{r}(x) \cap U_{r}(y)\right| \geq 2 a\left|U_{r}(x)\right| .
$$

For $x \in P$, set $V_{i}=\left|U_{1 / i}(x)\right|$, and

$$
\psi_{i}(x, \eta)=\left|U_{1 / i}(x)-H(x, \eta)\right|,
$$

$$
\phi_{i}(x)=\text { g.l.b. } S_{\eta}\left[\psi_{i}(x, \eta)<a V_{i}\right] .
$$


Since $e(x, y)$ is measurable in the pair of variables $x, y$, it follows that $\psi_{i}(x, \eta)$ is measurable for fixed $\eta$. Also, as a function of $\eta, \psi_{i}(x, \eta)$ is monotone and continuous on the left; hence

$$
\phi_{i}(x)<\zeta \text { if and only if } \psi_{i}(x, \zeta)<a V_{i} .
$$

Therefore $\phi_{i}$ is measurable.

Let $Q_{1}$ be the set of points where $f$ is a.t.d.; then $f_{1}, \cdots, f_{n}$ are defined in $Q_{1}$. Given $x \in Q_{1}$ and $\epsilon^{\prime}>0$, we may choose $\delta>0$ so that

$$
\psi_{i}\left(x, \epsilon^{\prime}\right)<a V_{i} \quad \text { if } \quad 1 / i<\delta \text {; }
$$

using (2.6) shows that

$$
\lim _{i \rightarrow \infty} \phi_{i}(x)=0, \quad x \in Q_{1} .
$$

By Lusin's and Egeroff's theorems, there is a closed set $Q \subset Q_{1}$ such that $\left|Q_{1}-Q\right|<\epsilon$, the $f_{k}$ are continuous in $Q$, and $\phi_{i}(x) \rightarrow 0$ uniformly in $Q$. We now prove that for each $\epsilon^{\prime}>0$ there is a $\delta>0$ such that

$$
e(x, y)<\epsilon^{\prime} \quad \text { if } \quad x, y \in Q, \quad|y-x|<\delta .
$$

Setting $\epsilon_{1}=\epsilon^{\prime} / 6$, we may choose $\delta$ so that

$$
\begin{gathered}
|F(y) \cdot v-F(x) \cdot v| \leq \epsilon_{1}|v| \quad \text { if } \quad x, y \in Q, \quad|y-x|<2 \delta, \\
\phi_{i}(x)<\epsilon_{1} \quad \text { if } \quad x \in Q, \quad 1 /(i+1)<\delta .
\end{gathered}
$$

Now take any $x, y \in Q$ with $|y-x|<\delta$. Let $j$ be the largest integer such that $1 / j \geq|y-x|$, and set

$$
R=U_{1 / j}(x) \cap U_{1 / j}(y) ; \quad \text { then }|R| \geq 2 a V_{j} .
$$

Since $1 /(j+1)<|y-x|<\delta,(2.10)$ and (2.6) give

$$
\psi_{j}\left(x, \epsilon_{1}\right), \quad \psi_{j}\left(y, \epsilon_{1}\right)<a V_{j} .
$$

Hence there is a point $z$ in $R$ in neither corresponding set; that is,

$$
|z-x|, \quad|z-y|<1 / j ; \quad e(x, z), \quad e(y, z)<\epsilon_{1} .
$$

Since $F(x) \cdot v$ is linear in $v$ and $|z-x|,|z-y|<2|y-x|$, we have

$$
\begin{aligned}
e(x, y)|y-x|= & |f(y)-f(x)-F(x) \cdot(y-x)| \\
\leq & |f(z)-f(x)-F(x) \cdot(z-x)| \\
& +|f(z)-f(y)-F(y) \cdot(z-y)|+|[F(y)-F(x)] \cdot(z-y)| \\
\leq & \epsilon_{1}[|z-x|+|z-y|+|z-y|]<\epsilon^{\prime}|y-x|
\end{aligned}
$$


if $y \neq x$, proving (2.8).

This fact, together with the continuity of the $f_{k}(x)$ in $Q$, shows that $f$ is "of class $C^{1}$ in terms of the $f_{k}$ in $Q$ ", as defined in [7] (the definition is given after (6.3), below). Hence, by [7, Lemma 2], we may extend $f$ to be smooth in $E^{n}$, completing the proof. (The extension is described in Section 4, below; by use of the results of that section, it is not hard to show that $f$ has the required properties.)

3. The theorem for unbounded sets. We remove the restriction of boundedness in Theorem 1, and give more information about the set in which $f$ may be left unaltered.

THEOREM 2. Let $A_{1}, A_{2}, \cdots$ be open sets in $E^{n}$ such that each has points in common with, at most, a finite number of the others, and let $\epsilon_{1}, \epsilon_{2}, \cdots$ be positive numbers. Let $K$ be the set of points $x$ such that there is a sequence of distinct sets $A_{\mu_{1}}, A_{\mu_{2}}, \cdots$ and a sequence of points $x_{1}, x_{2}, \cdots$ with $x_{i} \in A_{\mu_{i}}$ and $x_{i} \rightarrow x$. Let $P$ be a measurable subset of $E^{n}-K$, and let $f$ be a.t.d.a.e. in $P$ in terms of the $f_{k}$. Then there is a set $Q \subset P$ such that $Q$ is closed in $E^{n}-K$ and $\left|(P-Q) \cap A_{i}\right|<\epsilon_{i}$, and there is a smooth function $g$ in $E^{n}-K$ such that $g(x)=f(x)$ and $\partial g(x) / \partial x_{k}=f_{k}(x)$ in $Q$.

Remarks. Clearly $K$ is closed and $K \cap A_{i}=0$ for all $i$. If $Q^{*} \subset P, Q^{*}$ is closed in $E^{n}-K$, and for some positive continuous functions $\delta_{1}(x), \delta_{2}(x), \cdots$ in $Q^{*}$,

$$
e(x, y) \leq 1 / 2^{i} \quad \text { if } x \in Q^{*}, \quad|y-x|<\delta_{i}(x),
$$

the proof shows that we may make $Q \supset Q^{*}$. For instance, we may make $Q$ contain any given set of points of $P$ in which $f$ is totally differentiable and which has no accumulation points in $E^{n}-K$. On the other hand, we must expect to drop out a neighborhood of the set of points where $f$ is not totally differentiable. Further, we c annot in general keep in $Q$ any given closed set where $f$ is approximately totally differentiable, as is shown by the following example (in one variable):

$$
f(t)=t^{2} \sin (i / t)(t \neq 0), f(0)=0 \text {. }
$$

Proof of Theorem 2. For each pair $(k, l)$ of positive integers, let $U_{k, l}$ be the set of points $x$ satisfying the conditions (with a fixed $x_{0}$ in $E^{n}$ )

$$
k-1<\left|x-x_{0}\right|<k+1, \quad 1 /(l-1)>\operatorname{dist}(x, K)>1 /(l+1) ;
$$

for $k=1$ or $l=1$, we drop out the first inequalities. If $K$ is void, the index $l$ is 
not needed, and the situation is simpler. The $U_{k, l}$ are bounded open sets covering $E^{n}-K$, and each one touches at most eight others. Arrange them in a sequence $U_{1}^{\prime}, U_{2}^{\prime}, \cdots$.

For each $i$, let $\lambda_{i, 1}, \lambda_{i, 2}, \cdots$ be the (finite or infinite) set of numbers such that $U^{\prime} \lambda_{i, k} \cap A_{i} \neq 0$. Since the $\bar{U}_{j}^{\prime}$ are compact and in $E^{n}-K$, each touches at most a finite number of the $A_{i}$; hence for given $j$, there is at most a finite number of values of $i$ such that $\lambda_{i, k}=j$ for some $k$. Let $\epsilon_{j}^{\prime}$ be the smallest of the numbers $\epsilon_{i} / 2^{k}$, using these values of $i$ and corresponding $k$.

Considering $f$ and the $f_{k}$ in $P \cap U_{j}^{\prime}$ alone, apply the proof of Theorem 1 to find a closed set $Q_{j} \subset P \cap U_{j}^{\prime}$ such that $\left|P \cap U_{j}^{\prime}-Q_{j}\right|<\epsilon_{j}^{\prime}$, and such that $f$ is of class $C^{1}$ in terms of the $f_{k}$ in $Q_{j}$. Set

$$
V_{j}=U_{j}^{\prime}-Q_{j}, \quad V=U_{j} V_{j}, \quad Q=E^{n}-K-V .
$$

Then $V$ is open, $Q$ is closed in $E^{n}-K$, and $Q \cap U_{j}^{\prime} \subset Q_{j}$. Now

$$
\begin{aligned}
(P-Q) \cap A_{i}=P \cap V \cap A_{i} & =\mathrm{U}_{j}\left(P \cap V_{j} \cap A_{i}\right), \\
\left|P \cap V_{j}\right| & =\left|P \cap U_{j}^{\prime}-Q_{j}\right|<\epsilon_{j}^{\prime} .
\end{aligned}
$$

Since $V_{j} \subset U_{j}^{\prime}, P \cap V_{j} \cap A_{i}$ is void unless $j=\lambda_{i, k}$ for some $k$. Hence

$$
\left|(P-Q) \cap A_{i}\right| \leq \sum_{j}\left|P \cap V_{j} \cap A_{i}\right|<\sum_{k} \epsilon^{\prime} \lambda_{i, k} \leq \sum_{k} \epsilon_{i} / 2^{k}=\epsilon_{i} .
$$

Since each $U_{j}^{\prime}$ is open and $Q \cap U_{j}^{\prime} \subset Q_{j}, f$ is clearly of class $C^{1}$ in terms of the $f_{k}$ in $Q$. Hence, as before, we may extend the values of $f$ in $Q$ through $E^{n}-K$, as required. (We are applying $[7$, Lemma 2$]$ in an open set; the change required in the proof is very simple. Or we could use [7, Theorem III ].)

4. The theorem for Lipschitz functions. The following theorem has two parts, corresponding to the two theorems above.

THEOREM 3. For each positive integer $n$ there is a number $\rho_{n}$ (we may take $\left.\rho_{1}=1\right)$ with the following properties.

(a) Let $f$ be defined and satisfy a Lipschitz condition in the bounded closed set $P \subset E^{n}$ :

$$
|f(y)-f(x)| \leq N|y-x|, \quad x, y \in P .
$$

Then for each $\epsilon>0$ there is a closed set $Q \in P$ such that $|P-Q|<\epsilon$, and there is a smooth function $g$ in $E^{n}$ satisfying a Lipschitz condition (see (4.15)) with the constant $\rho_{n} N$, such that $g=f$ in $Q$. 
(b) Let the $A_{i}, \epsilon_{i}$, and $K$ be as in Theorem 2. Let $P$ be closed in $E^{n}-K$ (it may have accumulation points in K). Let $f$ be defined in $P$ and satisfy (4.1). Then there is a set $Q \subset P$ which is closed in $P$ (and hence in $E^{n}-K$ ) such that $\left|(P-Q) \cap A_{i}\right|<\epsilon_{i}$; and there is a function $g$ satisfying (4.15) in $E^{n}$ which is smooth in $E^{n}-Q^{*}$, where $Q^{*}=\bar{Q}-Q$, such that $g=f$ in $Q$.

(c) We may take $Q$ [in either (a) or (b)] so that $f$ is totally differentiable in $Q$ in terms of functions $f_{1}, \cdots, f_{n}$; we may then take $g$ so that $\partial g / \partial x_{k}=f_{k}$ in $Q(k=1, \cdots, n)$.

(d) Given a positive continuous function $\eta(x)$ in $E^{n}-K\left[\right.$ in $E^{n}$, for case (a) ], we may make

$$
|g(x)-f(x)|<\eta(x),
$$$$
x \in P \text {. }
$$

REMARKS. It is no restriction to take $P$ closed (or closed in $E^{n}-K$ ). For if $P$ is not closed, it is easily seen that we may extend $f$ (uniquely) over $\bar{P}$ so that it is continuous there; then (4.1) now holds in $\bar{P}$. (We can in fact extend $f$ to satisfy (4.1) in $E^{n}$; see [3] or [4].) Note that, in (b), $Q^{*} \subset \bar{P}-P$; if $K$ is void, then $Q^{*}$ is void, and $g$ is smooth in $E^{n}$. As an immediate consequence of (4.15), we have

$$
\left|\sum v_{k} \partial_{g}(x) / \partial x_{k}\right| \leq \rho_{n} N \quad \text { if } \quad x \in E^{n}-Q^{*},|v|=1 .
$$

The hypothesis of total differentiability a.e. in $P$, together with

$$
\left|\Sigma v_{k} f_{k}(x)\right| \leq N|v|
$$

where the $f_{k}$ are defined, is not enough to give the theorem (unless, for instance, $P=E^{n}$ ), as simple examples show. (Compare the examples in H. Whitney [8].) If we wish to prove (4.3) rather than (4.15), the proof may be slightlysimplified; of course (4.15) follows from (4.3) if $Q^{*}=0$ (hence if $K=0$ ). See also the remarks following Theorem 2.

Proof of Theorem 3. To prove the theorem, we first note that (a) is contained in (b); use $A_{1}=E^{n}, \epsilon_{1}=\epsilon$. Next, (d) will follow at once from (4.1) and (4.15) if we make sure that each point of $P$ is sufficiently close to some point of $Q$; this will clearly be the case if, in applying the proof of Theorem 2, we take the $\epsilon_{j}^{\prime}$ small enough. Also, just as in Theorem 2, (c) will hold. It remains to show that we can obtain the properties in (b), using the proof of Theorem 2. We do this here, except for showing that we can make $\rho_{1}=1$.

We must examine the proof of $[7$, Lemma 2$]$. First, since $f$ is totally differentiable a.e. in $P[6, \mathrm{p} .311]$, we may choose $Q$ as in the proof of Theorem 2; 
recall that the $f_{k}$ are continuous in $Q$. We shall use a cubical subdivision of $E^{n}-\bar{Q}$, essentially as in [7]. For each integer $s$ (in [7], only $s \geq 0$ was used), let $K_{s}^{\prime}$ be the set of all cubes of edge length $1 / 2^{s}$, the coordinates of whose corners are integral multiples of $1 / 2^{s}$. Let $K_{s}^{\prime \prime}$ consist of the cubes of $K_{s}^{\prime}$ whose distances from $\bar{Q}$ are at least $6 n^{1 / 2} / 2^{s}$. Let $K_{s}$ consist of the cubes of $K_{s}^{\prime \prime}$ which are not in cubes of $K_{s-1}^{\prime \prime}$. Take any cube $C \in K_{s}$; suppose $C \subset C^{\prime}$, $C^{\prime} \in K_{s-1}^{\prime}$. Then $\operatorname{dist}\left(C^{\prime}, \bar{Q}\right)<6 n^{1 / 2} / 2^{s-1}$. Therefore, clearly

$$
6 n^{1 / 2} / 2^{s} \leq \operatorname{dist}(C, \bar{Q})<13 n^{1 / 2} / 2^{s},
$$

Take $C \in K_{s}, C^{\prime} \in K_{s+2}$. Then each point of $C^{\prime}$ is within

$$
n^{1 / 2} / 2^{s+2}+13 n^{1 / 2} / 2^{s+2}
$$

from $\bar{Q}$; hence

$$
\operatorname{dist}\left(C, C^{\prime}\right) \geq(5 / 2) n^{1 / 2} / 2^{s}, \quad G \in K_{s}, \quad C^{\prime} \in K_{s+2} .
$$

Let $y^{1}, y^{2}, \cdots$ be the set of all corners of all cubes of all $K_{s}$. Choose $x^{\nu} \in \bar{Q}$ with $\left|x^{\nu}-y^{\nu}\right|=\operatorname{dist}\left(y^{\nu}, \bar{Q}\right)$. Let $b_{\nu}$ be the largest length of edge of any cube of any $K_{s}$ with $y^{\nu}$ as a corner, and let $I_{\nu}$ be the cube defined by

$$
\left|x_{i}-y_{i}^{\nu}\right| \leq b_{\nu}(i=1, \cdots, n) \text {. }
$$

Let $\phi_{0}^{\prime}$ be a smooth function which is positive within a fixed unit cube and is zero outside; by a translation and similarity transformation, define $\phi_{\nu}^{\prime}$, positive within $I_{\nu}$ and zero outside. Set $\phi_{\nu}=\phi_{\nu}^{\prime} / \Sigma \phi^{\prime} \lambda$; then $\phi_{\nu}$ is positive within $I_{\nu}$ and zero outside, and $\Sigma \phi_{\nu}=1$ in $E^{n}-\bar{Q}$. Since there is at most some fixed number of shapes of cubes (of some $K_{s}$, and $K_{s+1}$ perhaps) forming any $I_{\nu}$, there is clearly a number $M_{n} \geq 1$ with the following property (compare [7, Section 10]): taking $|v|=1$,

$$
\left|\Sigma v_{i} \partial \phi_{\nu} / \partial x_{i}\right|<2^{s} M_{n} \text { if } \phi_{\nu}(y) \neq 0 \text { for some } y \in C \in K_{s} \text {. }
$$

Extend $f$ to be continuous in $\bar{P}$ (if $\bar{P} \neq P$ ); (4.1) still holds. For any $x^{*} \in \bar{Q}$ and any $x \in E^{n}$, set

$$
\psi\left(x ; x^{*}\right)=f\left(x^{*}\right)+\sum f_{i}\left(x^{*}\right)\left(x_{i}-x_{i}^{*}\right) ;
$$

this is the value at $x$ of the linear function approximating to $f$ at $x^{*}$. Then set

$$
g(x)=\sum \phi_{\nu}(x) \psi\left(x ; x^{\nu}\right), \quad x \in E^{n}-\bar{Q} .
$$


It is not hard to show that if $g=f$ in $\bar{Q}$, then $g$ is smooth in $E^{n}-Q^{*}$ and $\partial g / \partial x_{i}=f_{i}$ in $Q$; see the proof of [7, Lemma 2]. We must still prove (4.15). Take first any $x$ and $x^{\prime}$ in $E^{n}-\bar{Q}$; say for definiteness that

$$
x \in C \in K_{s}, \quad x^{\prime} \in C^{\prime} \in K_{s^{\prime}}, \quad s \geq s^{\prime} .
$$

Let $x^{*}$ be a point of $\bar{Q}$ nearest to $x$. Since $\Sigma \phi_{\nu}(x)=1$, we may write

$$
g(x)=f\left(x^{*}\right)+\sum_{\nu} \phi_{\nu}(x)\left[f\left(x^{\nu}\right)-f\left(x^{*}\right)\right]+\sum_{\nu, i} \phi_{\nu}(x) f_{i}\left(x^{\nu}\right)\left(x_{i}-x_{i}^{\nu}\right) .
$$

Hence

$$
\begin{gathered}
g\left(x^{\prime}\right)-g(x)=\sum_{\nu}\left[\phi_{\nu}\left(x^{\prime}\right)-\phi_{\nu}(x)\right]\left[f\left(x^{\nu}\right)-f\left(x^{*}\right)\right] \\
+\sum_{\nu, i}\left[\phi_{\nu}\left(x^{\prime}\right)-\phi_{\nu}(x)\right] f_{i}\left(x^{\nu}\right)\left(x_{i}^{\prime}-x_{i}^{\nu}\right)+\sum_{\nu, i} \phi_{\nu}(x) f_{i}\left(x^{\nu}\right)\left(x_{i}^{\prime}-x_{i}\right) .
\end{gathered}
$$

We shall find a bound for each non-zero term. First we show that

$$
\left|\phi_{\nu}\left(x^{\prime}\right)-\phi_{\nu}(x)\right|\left|f\left(x^{\nu}\right)-f\left(x^{*}\right)\right| \leq 64 N M_{n} n^{1 / 2}\left|x^{\prime}-x\right| .
$$

Consider first any $\nu$ such that $\phi_{\nu}(x) \neq 0$. Then by (4.6),

$$
\left|\phi_{\nu}\left(x^{\prime}\right)-\phi_{\nu}(x)\right|<2^{s} M_{n}\left|x^{\prime}-x\right| \text {. }
$$

Also, since

$$
\begin{aligned}
\left|x^{*}-x\right| & \leq \operatorname{diam}(C)+\operatorname{dist}(C, \bar{Q}) \leq 14 n^{1 / 2} / 2^{s}, \\
\left|y^{\nu}-x\right| & \leq 2 \operatorname{diam}(C) \leq 2 n^{1 / 2} / 2^{s}, \\
\left|y^{\nu}-x^{\nu}\right| & \leq\left|x^{*}-y^{\nu}\right| \leq 16 n^{1 / 2} / 2^{s},
\end{aligned}
$$

we have

$$
\left|x^{\nu}-x^{*}\right| \leq 32 n^{1 / 2} / 2^{s}
$$

and hence

$$
\left|f\left(x^{\nu}\right)-f\left(x^{*}\right)\right| \leq 32 N n^{1 / 2} / 2^{s} .
$$


These relations give (4.11). Next consider any $\nu$ such that $\phi_{\nu}\left(x^{\prime}\right) \neq 0$. Then (using inequalities like those above) we obtain

$$
\begin{aligned}
\left|x^{\nu}-x^{*}\right| & \leq\left|x^{\nu}-x^{\prime}\right|+\left|x^{\prime}-x\right|+\left|x-x^{*}\right| \\
& \leq 18 n^{1 / 2} / 2^{s^{\prime}}+\left|x^{\prime}-x\right|+14 n^{1 / 2} / 2^{s} \leq 32 n^{1 / 2} / 2^{s^{\prime}}+\left|x^{\prime}-x\right| .
\end{aligned}
$$

In the present case, (4.12) holds with $s^{\prime}$. Suppose first that

$$
\left|x^{\prime}-x\right| \leq 32 n^{1 / 2} / 2^{s^{\prime}} \text {. }
$$

Then

$$
\left|f\left(x^{\nu}\right)-f\left(x^{*}\right)\right| \leq N \cdot 64 n^{1 / 2} / 2^{s^{\prime}},
$$

and (4.11) follows. If $\left|x^{\prime}-x\right|>32 n^{1 / 2} / 2^{s^{\prime}}$, then $\left|x^{\nu}-x^{*}\right|<2\left|x^{\prime}-x\right|$, and since $\left|\phi_{\nu}\left(x^{\prime}\right)-\phi_{\nu}(x)\right| \leq 2$ and $4 N<64 N M_{n} n^{1 / 2}$, (4.11) follows again.

Next we show that

$$
\left|\phi_{\nu}\left(x^{\prime}\right)-\phi_{\nu}(x)\right|\left|f_{i}\left(x^{\nu}\right)\right|\left|x_{i}^{\prime}-x_{i}^{\nu}\right| \leq 40 N M_{n} n^{1 / 2}\left|x^{\prime}-x\right| .
$$

We may suppose that $\phi_{\nu}\left(x^{\prime}\right) \neq 0$, in which case $\left|x^{\prime}-x^{\nu}\right| \leq 18 n^{1 / 2} / 2^{s^{\prime}}$, or $\phi_{\nu}(x) \neq 0$, in which case $\left|x^{\prime}-x^{\nu}\right| \leq 18 n^{1 / 2} / 2^{s}+\left|x^{\prime}-x\right|$; in either case,

$$
\left|x_{i}^{\prime}-x_{i}^{\nu}\right| \leq\left|x^{\prime}-x^{\nu}\right| \leq 18 n^{1 / 2} / 2^{s^{\prime}}+\left|x^{\prime}-x\right| \text {. }
$$

First suppose that $\left|x^{\prime}-x\right| \leq 2 n^{1 / 2} / 2^{s^{\prime}}$. Then, by (4.5), $s \leq s^{\prime}+1$. Hence, using (4.6) with $s$ or $s^{\prime}$ we get

$$
\left|\phi_{\nu}\left(x^{\prime}\right)-\phi_{\nu}(x)\right| \leq 2^{s^{\prime}+1} M_{n}\left|x^{\prime}-x\right| ;
$$

since $\left|f_{i}\left(x^{\nu}\right)\right| \leq N,(4.13)$ follows. Next suppose that $\left|x^{\prime}-x\right|>2 n^{1 / 2} / 2^{s^{\prime}}$. Then $\left|\phi_{\nu}\left(x^{\prime}\right)-\phi_{\nu}(x)\right| \leq 2$, and $\left|x_{i}^{\prime}-x_{i}^{\nu}\right|<10\left|x^{\prime}-x\right|$, giving (4.13) again. Finally, we have

$$
\left|\phi_{\nu}(x) f_{i}\left(x^{\nu}\right)\left(x_{i}^{\prime}-x_{i}\right)\right| \leq N\left|x^{\prime}-x\right| .
$$

There is clearly a number $c_{n}$ such that for any $x$, there are at most $c_{n}$ values 
of $\nu$ such that $\phi_{\nu}(x) \neq 0$. In the three groups of terms in (4.10), there are at most $2 c_{n}, 2 c_{n} n$, and $c_{n} n$ non-zero terms respectively. Hence, by (4.11), (4.13) and (4.14), we have

$$
\left|g\left(x^{\prime}\right)-g(x)\right| \leq c_{n} N M_{n} n^{1 / 2}(128+80 n+n)\left|x^{\prime}-x\right|,
$$

which gives

$$
\left|g\left(x^{\prime}\right)-g(x)\right| \leq \rho_{n} N\left|x^{\prime}-x\right|, \quad \rho_{n}=209 c_{n} M_{n} n^{3 / 2} .
$$

If $x$ and $x^{\prime}$ are in $\bar{Q},(4.15)$ follows from (4.1), since $g=f$ (or the extended $f$ ) in $\bar{Q}$. Suppose finally that $x \in \bar{Q}, x^{\prime} \in E^{n}-\bar{Q}$ (or vice versa). Let $x^{\prime \prime}$ be the last point of the segment $x x^{\prime}$ in $\bar{Q}$. Then (4.1) holds for $x$ and $x^{\prime \prime}$, and (4.15) holds for $x^{\prime \prime \prime}$ and $x^{\prime}$, with $x^{\prime \prime \prime}$ in $x^{\prime \prime} x^{\prime}$ and arbitrarily close to $x^{\prime \prime}$; hence (4.15) holds in all cases, and the proof is complete.

5. Lipschitz functions of one variable. We must prove Theorem 3, with $n=1$, $\rho_{1}=1$. The proof is elementary in nature; we do not need [7]. Find a closed subset $Q_{1}$ of $E^{1}-K$ (or of $E^{1}$, in case (a)) as in the proof of Theorem 2 (or Theorem 1, if we are only using (a)). Now (4.1) holds in $Q_{1}, f_{1}$ is continuous in $Q_{1}$, and $f$ is smooth in terms of $f_{1}$ in $Q_{1}$ (see Section 3, above), that is, for each $x \in Q_{1}$ and each $\epsilon^{\prime}>0$ there is a $\delta>0$ such that

$$
\begin{aligned}
\left|f\left(x^{\prime \prime}\right)-f\left(x^{\prime}\right)-\left(x^{\prime \prime}-x^{\prime}\right) f_{1}\left(x^{\prime}\right)\right| & \leq \epsilon^{\prime}\left|x^{\prime \prime}-x^{\prime}\right| \\
& \text { if }\left|x^{\prime \prime}-x\right|, \quad\left|x^{\prime}-x\right|<\delta, \quad x^{\prime}, x^{\prime \prime} \in Q_{1} .
\end{aligned}
$$

Let primes on functions denote differentiation. We shall find a set $Q$ which is closed in $E^{1}-K$, with $\left|Q_{1}-Q\right|<\epsilon^{*}$ in case (a) or $\left|\left(Q_{1}-Q\right) \cap A_{i}\right|<\epsilon_{i}^{*}$ in case (b), and a function $g$ which satisfies (4.1) in $E^{1}$ and is smooth in $E^{1}-K$, and such that $g=f$ and $g^{\prime}=f_{1}$ in $Q$; for $\epsilon^{*}$ or the $\epsilon_{i}^{*}$ small enough, $Q$ and $g$ have the required properties.

Let $I_{1}, I_{2}, \cdots$ be the closed intervals whose interiors fill out $E^{1}-Q_{1} \cup K$. Extend $f$ through $E^{1}$ so that (4.1) holds there; see [3] or [4]. Set $g_{0}=f$ in $Q_{1} \cup K$, and let $g_{0}$ be linear in the $I_{k}$, so that $g_{0}$ is continuous in the closed intervals. Then $g_{0}$ is continuous in $E^{1}$, and satisfies (4.1) there.

We shall need the following lemma.

Lemma 1. Let $\phi$ be defined and satisfy (4.1) in the closed interval $\left[a^{*}, b\right]$, and let $\phi b$ be linear in the subinterval $[a, b]$. Then there is an arbitrarily small 
interval $\left[a^{\prime}, a^{\prime \prime}\right]$ about a such that $\phi^{\prime}\left(a^{\prime}\right)$ exists, and there is a function $\psi$ in $\left[a^{*}, b\right]$ which equals $\phi$ in $\left[a^{*}, a^{\prime}\right] \cup\left[a^{\prime \prime}, b\right]$ and is smooth in $\left[a^{\prime}, b\right]$, and is such that $\psi^{\prime}\left(a^{\prime}\right)=\phi^{\prime}\left(a^{\prime}\right)$, and for $x$ in $\left[a^{\prime}, a^{\prime \prime}\right], \psi^{\prime}(x)$ lies between $\phi^{\prime}\left(a^{\prime}\right)$ and $\phi^{\prime}\left(a^{\prime \prime}\right)$.

We use the notation $\Delta \phi(x, y)=[\phi(y)-\phi(x)] /(y-x)$. If $\phi$ is linear in some interval $\left[x_{0}, b\right]$ with $x_{0}<a$, we may set $\psi=\phi$. If not, we may choose $c<a$. arbitrarily close to $a$ so that $\Delta \phi(c, a) \neq \Delta \phi(a, b)$. Suppose for definiteness that $\Delta \phi(c, a)<\Delta \phi(a, b)$. Take $a^{\prime \prime}>a$ arbitrarily close to $a$. Because of (4.1), $\phi$ is absolutely continuous, and $\phi\left(a^{\prime \prime}\right)-\phi(c)=\int_{c}^{a^{\prime \prime}} \phi^{\prime}(x) d x$. Hence there is a point $a^{\prime}$ in $[c, a]$ such that $\phi^{\prime}\left(a^{\prime}\right)$ exists and

$$
\phi^{\prime}\left(a^{\prime}\right)<\Delta \phi\left(a^{\prime}, a^{\prime \prime}\right)<\Delta \phi\left(a^{\prime \prime}, b\right)=\phi^{\prime}\left(a^{\prime \prime}\right) ;
$$

that is, the tangents at $a^{\prime}$ and $a^{\prime \prime}$ intersect at a point $x^{\prime}$ between $a^{\prime}$ and $a^{\prime \prime}$. Using these tangents except near $x^{\prime}$, and smoothing near $x^{\prime}$, gives the required $\psi$.

We return to the theorem. Let $x_{1}, x_{2}, \cdots$ be the set of end points of the intervals $I_{k}$. Let $I_{1}^{\prime \prime}$ be an interval about $x_{1}$, of length $<\epsilon_{1}^{\prime \prime}$ for some $\epsilon_{1}^{\prime \prime}$ (see below), with one end point interior to an interval $I_{k}$ with $x_{1}$ as end point. Apply the lemma (or the lemma with $x$ replaced by $-x$ ) to find an interior interval $I_{1}^{\prime}$ $=\left[a_{1}^{\prime}, a_{1}^{\prime \prime}\right]$ about $x_{1}$, and using $\phi=g_{0}$ in $I_{1}^{\prime \prime}$, define $\psi=g$ in $I_{1}^{\prime}$. We may require that neither $a_{1}^{\prime}$ nor $a_{1}^{\prime \prime}$ is any $x_{i}$. In general, having found disjoint intervals $I_{1}{ }^{\prime}, \cdots, l_{j-1}{ }^{\prime}$, let $x_{h}$ be the first point of the sequence which is in none of these, and let $l_{j}^{\prime \prime}$ be an interval about $x_{h}$, of length $<\epsilon_{j}^{\prime \prime}$, disjoint from the preceding $l_{i}{ }^{\prime}$. Apply the lemma as before to find $l_{j}^{\prime}$, and define $g$ in $l_{j}^{\prime}$. Set $g=g_{0}$ elsewhere in $E^{1}$. Let $Q$ be the set of points of $Q_{1}$ interior to no $I_{j}^{\prime}$. For small enough $\epsilon_{j}^{\prime \prime}$, the inequalities with $\epsilon^{*}$ or $\epsilon_{i}^{*}$ hold. We shall show that $g$ is smooth in $E^{1}-K$ and $g^{\prime}=f_{1}$ in $Q$; the other properties of $g$ are clear.

Clearly $g^{\prime}$ is continuous in a neighborhood of any point interior to an $l_{j}^{\prime}$ or an $I_{k}$, that is, in $E^{1}-K \cup Q$. Now take any $x \in Q$; we shall show that $g^{\prime}(x)$ $=f_{1}(x)$ and $g^{\prime}$ is continuous at $x$, considering only points $x^{\prime} \geq x$ for which $g^{\prime}\left(x^{\prime}\right)$ is defined. The same fact holds for $x^{\prime} \leq x$, and this will complete the proof. By definition of $g$, this is true if $x$ is the left hand end point of some $l_{j}{ }^{\prime}$; suppose this is not the case.

Given $\epsilon^{\prime}>0$, choose $\delta$ so that (5.1) holds, and so that $\left|f_{1}\left(x^{\prime}\right)-f_{1}(x)\right|<\epsilon^{\prime}$ for $x^{\prime} \in Q_{1},\left|x^{\prime}-x\right|<\delta$. Choose $y>x$ in $Q_{1}$ within $\delta$ of $x$. Now any difference quotient of $f$, with points in $[x, y] \cap Q_{1}$, is within $2 \epsilon^{\prime}$ of $f_{1}(x)$; hence 
clearly any difference quotient of $g_{0}$ in $[x, y]$ is within $2 \epsilon^{\prime}$ of $f_{1}(x)$. Hence, for any $x^{\prime}$ in $[x, y]$ such that $g_{0}^{\prime}\left(x^{\prime}\right)$ exists, $\left|g_{0}^{\prime}\left(x^{\prime}\right)-f_{1}(x)\right|<2 \epsilon^{\prime}$. Because of the last property in the lemma, $\left|g^{\prime}\left(x^{\prime}\right)-f_{1}(x)\right|<2 \epsilon^{\prime}$ if $g^{\prime}\left(x^{\prime}\right)$ exists. Since $g\left(x^{\prime}\right)-g(x)=\int_{x}^{x^{\prime}} g^{\prime}(t) d t$, this shows that $g^{\prime}(x)$ exists (as a right hand derivative) and equals $f_{1}(x)$, and proves the required continuity.

6. Functions with totally differentiable $m$ th partial derivatives. We shall prove a theorem corresponding to $(a) \longrightarrow(c)$ in Theorem 1 ; the extension to the case corresponding to Theorem 2 is clear.

THEOREM 4. Let $f$ and its partial derivatives of order $\leq m$ be defined in a bounded open set $P \subset E^{n}$, and let each mth partial derivative be totally differentiable a.e. in $P$. Then for each $\epsilon>0$ there is a closed set $Q \subset P$ such that $|P-Q|<\epsilon$, and there is a function $g$ with continuous $(m+1)$ th partial derivatives in $E^{n}$ such that all partial derivatives of $f$ of order $\leq m+1$ exist in $Q$ and equal those of $g$ there. In particular, $g=f$ in $Q$.

Because of Theorem 1, we may suppose $m \geq 1$. We use the notation of [7]; thus

$$
\frac{f_{k}(x)}{k !}\left(x^{\prime}-x\right)^{k}=\frac{f_{k_{1}} \cdots k_{n}\left(x_{1}, \cdots, x_{n}\right)}{k_{1} ! \cdots k_{n} !}\left(x_{1}^{\prime}-x_{1}\right)^{k_{1}} \cdots\left(x_{n}^{\prime}-x_{n}\right)^{k_{n}}
$$

[do not confuse with the earlier $\left.f_{k}(x)\right], \sigma_{k}=k_{1}+\cdots+k_{n}$, and so on. Also

$$
f_{k_{1}} \cdots k_{n}=\partial^{\sigma_{k}} f / \partial x_{1}^{k_{1}} \cdots \partial x_{n}^{k_{n}}
$$

where defined.

Take any $k$ with $\sigma_{k}=m-1$, and any integers $i$ and $j$. Since $\partial f_{k} / \partial x_{i}$ and $\partial f_{k} / \partial x_{j}$ are defined in $P$ and are totally differentiable a.e. in $P$, it follows that their partial derivatives $\partial^{2} f_{k} / \partial x_{i} \partial x_{j}$ and $\partial^{2} f_{k} / \partial x_{j} \partial x_{i}$ exist a.e. in $P$; by a theorem of Currier [1], these are equal a.e. in $P$. Where this is so for all $i, j$, it is clear that we may define $f_{k}$ with $\sigma_{k}=m+1$ uniquely. Let $P^{\prime}$ be the subset of $P$ in which the $f_{k}$ exist for $\sigma_{k} \leq m+1$, and each $f_{k}\left(\sigma_{k}=m\right)$ is totally differentiable in terms of the $f_{l}\left(l_{i} \geq k_{i}, \sigma_{l}=m+1\right)$; then $\left|P-P^{\prime}\right|=0$. As seen in Section 2, the $f_{k}$ are measurable.

As in [7], let $\psi_{k}\left(x^{\prime} ; x\right)$, for $\sigma_{k} \leq m$, be the value at $x^{\prime}$ of the polynomial of degree at most $m-\sigma_{k}$ which has the same value and partial derivatives of order $\leq m-\sigma_{k}$ at $x$ as $f_{k}$. Then

$$
\psi_{k}\left(x^{\prime} ; x\right)=\sum_{\sigma_{l} \leq m-\sigma_{k}} \frac{f_{k+l}(x)}{l !}\left(x^{\prime}-x\right)^{l} .
$$


Let $R_{k}\left(x^{\prime} ; x\right)$ be the corresponding remainder in Taylor's expansion:

$$
R_{k}\left(x^{\prime} ; x\right)=f_{k}\left(x^{\prime}\right)-\psi_{k}\left(x^{\prime} ; x\right), \quad \sigma_{k} \leq m .
$$

Define $\psi_{k}^{\prime}$ and $R_{k}^{\prime}$ similarly for $x \in P^{\prime}$, with $m$ replaced by $m+1$. We shall say a remainder $R_{k}^{\prime}$ is of order $m^{\prime}$ at $x^{0}$ if the following is true. For each $\epsilon^{\prime}>0$ there is a $\delta>0$ such that

$$
\left|R_{k}^{\prime}\left(x ; x^{0}\right)\right| \leq \epsilon^{\prime}\left|x-x^{0}\right|^{m^{\prime}} \quad \text { if } \quad\left|x-x^{0}\right|<\delta .
$$

Recall from [7] that in a closed set, $f$ is of class $C^{m+1}$ in terms of the $f_{k}\left(\sigma_{k}\right.$ $\leq m+1)$ if and only if each $R_{k}^{\prime}$ is of order $m+1-\sigma_{k}$ uniformly in a neighborhood of each point.

With the help of Lemma 2 below, we prove Theorem 4 as follows. By Lusin's Theorem, there is a closed set $Q^{\prime} \subset P^{\prime}$ with $\left|P^{\prime}-Q^{\prime}\right|<\epsilon / 2$ such that each $f_{k}(x)$ with $\sigma_{k}=m+1$ is continuous in $Q^{\prime}$; that is, $R_{k}^{\prime}\left(\sigma_{k}=m+1\right)$ is of order 0 in $Q^{\prime}$. For each integer $i$ and each $x^{0} \in Q^{\prime}$, let $\delta_{i}\left(x^{0}\right)$ be the upper bound of numbers $\delta \leq 1$ such that (6.3) holds with $m^{\prime}=m-\sigma_{k}+1, \epsilon^{\prime}=1 / 2^{i}$, for all $k$ with $\sigma_{k} \leq m+1$. Then by the lemma, $\delta_{i}(x)>0$ in $Q^{\prime}$. As in Section 2, we see that the $\delta_{i}(x)$ are measurable. Find sets $Q_{i}$ as in Section 2 , and set $Q=Q_{1} \cap Q_{2} \cap \cdots$. Then clearly $f$ is of class $C^{m+1}$ in $Q$ in terms of the $f_{k}$, and hence [7, Lemma 2] $f$ may be extended from $Q$ over $E^{n}$ so that

$$
\partial^{\sigma_{k}} f / \partial x_{1}^{k} \cdots \partial x_{n}^{k_{n}}=f_{k}
$$

in $Q$. This extension is the required $g$. There remains to prove

Lemma 2. Let $P$ be open, let

$$
\partial^{\sigma_{k}} f / \partial x_{1}^{k} \cdots \partial x_{n}^{k}=f_{k}
$$

in $P$ for $\sigma_{k} \leq m$, let $f_{k}\left(x^{0}\right)$ be defined for $\sigma_{k}=m+1$, and let the $f_{k}\left(\sigma_{k}=m\right)$ be totally differentiable in terms of the $f_{l}\left(l_{i} \geq k_{i}, \sigma_{l}=m+1\right)$ at $x^{0}$. Define $R_{k}^{\prime}\left(x ; x^{0}\right)$ as above. Then $R_{k}^{\prime}$ is of order $m-\sigma_{k}+1$ at $x^{0}$ if $\sigma_{k} \leq m-1$.

Note that the hypothesis shows that $R_{k}^{\prime}$ for $\sigma_{k}=m$ is of order 1 at $x^{0}$. Suppose we have proved Lemma 2 for the case that $f_{k}\left(x^{0}\right)=0$ for all $k$, $\sigma_{k} \leq m+1$. Then it holds for the general case. For set

$$
\bar{f}_{k}(x)=f_{k}(x)-\psi_{k}^{\prime}\left(x ; x^{0}\right)=R_{k}^{\prime}\left(x ; x^{0}\right) \quad\left(\sigma_{k} \leq m+1\right) ;
$$

then $\bar{f}_{k}\left(x^{0}\right)=0$. Also, since $\bar{\psi}_{k}^{\prime}\left(x ; x^{0}\right)=0$ (using the $\left.\bar{f}_{l}\right), \bar{R}_{k}^{\prime}\left(x ; x^{0}\right)=R_{k}^{\prime}\left(x ; x^{0}\right)$. 
Since the $\bar{R}_{k}^{\prime}$ are of order $m-\sigma_{k}+1$ at $x^{0}$ for $\sigma_{k}=m$, the lemma shows that this is true also for $\sigma_{k}<m$. Thus $R_{k}^{\prime}=\bar{R}_{k}^{\prime}$ is of order $m-\sigma_{k}+1$ at $x^{0}$, as required.

We shall need Taylor's Theorem with exact remainder:

LEMMA 3. Let $\phi$ be a function of one variable such that $\phi^{(h)}=d^{h} \phi / d x^{h}$ exists for $h \leq m^{\prime}$ in an interval and is bounded. Then

$$
\begin{aligned}
\phi\left(t_{1}\right)= & \sum_{h=0}^{m^{\prime}} \frac{\phi^{(h)}\left(t_{0}\right)}{h !}\left(t_{1}-t_{0}\right)^{h} \\
& +\frac{1}{\left(m^{\prime}-1\right) !} \int_{t_{0}}^{t_{1}}\left(t_{1}-s\right)^{m^{\prime}-1}\left[\phi^{\left(m^{\prime}\right)}(s)-\phi^{\left(m^{\prime}\right)}\left(t_{0}\right)\right] d s .
\end{aligned}
$$

Since $\phi^{\left(m^{\prime}\right)}$ is bounded, $\phi^{\left(m^{\prime}-1\right)}$ satisfies a Lipschitz condition; hence for any smooth $\alpha, \beta=\alpha \phi^{\left(m^{\prime}-1\right)}$ is absolutely continuous, and

$$
\int_{a}^{b}(d \beta / d t) d t=\beta(b)-\beta(a) \text {. }
$$

Therefore the usual proof applies.

We return to Lemma 2, assuming $f_{k}\left(x^{0}\right)=0\left(\sigma_{k} \leq m+1\right)$. Set

$$
x^{i}=\left(x_{1}, \cdots, x_{i}, x_{i+1}^{0}, \cdots, x_{n}^{0}\right) ;
$$

then $x^{n}=x$. Take any $i>0$, and any $k$ with $\sigma_{k} \leq m-1$. Set $m^{\prime}=m-\sigma_{k}$, $k(i)=\left(k_{1}, \cdots, k_{i}+m^{\prime}, \cdots, k_{n}\right)$, and

$$
x^{i}(s)=\left(x_{1}, \cdots, x_{i-1}, s, x_{i+1}^{0}, \cdots, x_{n}^{0}\right) .
$$

Then $x^{i}\left(x_{i}^{0}\right)=x^{i-1}, x^{i}\left(x_{i}\right)=x^{i}$. For some $\delta_{1}>0$, the $f_{k}(x)\left(\sigma_{k} \leq m\right)$ are bounded for $\left|x-x^{0}\right|<\delta_{1}$. Lemma 3 gives

$$
\begin{aligned}
f_{k}\left(x^{i}\right)= & \psi_{k}\left(x^{i} ; x^{i-1}\right) \\
& +\frac{1}{\left(m^{\prime}-1\right) !} \int_{x_{\imath}^{0}}^{x_{i}}\left(x_{i}-s\right)^{m^{\prime}-1}\left\{f_{k(i)}\left[x^{i}(s)\right]-f_{k(i)}\left(x^{i-1}\right)\right\} d s .
\end{aligned}
$$

Since $f_{l}\left(x^{i}(s)\right)=R_{l}^{\prime}\left(x^{i}(s) ; x^{0}\right)$, and so on, the definition of $R_{k}$ gives

$$
\begin{aligned}
R_{k}\left(x^{i} ; x^{i-1}\right)=\frac{1}{\left(m^{\prime}-1\right) !} \int_{x_{i}^{0}}^{x_{i}}\left(x_{i}-s\right)^{m^{\prime}-1} \\
\quad \times\left\{R_{k(i)}^{\prime}\left[x^{i}(s) ; x^{0}\right]-R_{k(i)}^{\prime}\left(x^{i-1} ; x^{0}\right)\right\} d s .
\end{aligned}
$$


For a certain $\epsilon^{*}$ chosen below, choose $\delta \leq \delta_{1}$ so that

$$
\left|R_{l}^{\prime}\left(x^{\prime} ; x^{0}\right)\right| \leq \epsilon^{*}\left|x^{\prime}-x^{0}\right| \quad \text { if } \sigma_{l}=m, \quad\left|x^{\prime}-x^{0}\right|<\delta .
$$

Then if $\left|x-x^{0}\right|<\delta$, using $\left|x^{i}(s)-x^{0}\right| \leq\left|x-x^{0}\right|$ for $x_{i}^{0} \leq s \leq x_{i}$, and so on, gives

$$
\left|R_{k}\left(x^{i} ; x^{i-1}\right)\right| \leq \frac{2 \epsilon^{*}\left|x-x^{0}\right|}{\left(m^{\prime}-1\right) !} \int_{x_{i}^{0}}^{x_{i}}\left(x_{i}-s\right)^{m^{\prime}-1} d s \leq \frac{2 \epsilon^{*}\left|x-x^{0}\right|^{\prime}+1}{m^{\prime} !} .
$$

Now in $[7,(6.3)]$, subtract $f_{k}\left(x^{\prime \prime}\right)$ from both sides, and change $x, x^{\prime}, x^{\prime \prime}$ to $x^{i-1}, x^{\imath}, x$ respectively; this gives

$$
R_{k}\left(x ; x^{i-1}\right)-R_{k}\left(x ; x^{i}\right)=\sum_{\sigma_{l} \leq m-\sigma_{k}} \frac{R_{k+l}\left(x^{i} ; x^{i-1}\right)}{l !}\left(x-x^{i}\right)^{l} .
$$

Hence,

$$
\left|R_{k}\left(x ; x^{i-1}\right)-R_{k}\left(x ; x^{i}\right)\right| \leq 2 \epsilon^{*}\left|x-x^{0}\right|^{m^{-} \sigma_{k}+1} \sum_{\sigma_{l} \leq_{m}-\sigma_{k}} \frac{1}{\left(m-\sigma_{k}-\sigma_{l}\right) ! l !} .
$$

Let $A_{k}$ denote the sum, and let $A$ be the largest $A_{k}$. Since $R_{k}^{\prime}\left(x ; x^{0}\right)=R_{k}\left(x ; x^{0}\right)$ for the case at hand, adding the inequalities for $i=1, \cdots, n$ gives

$$
\left|R_{k}^{\prime}\left(x ; x^{0}\right)\right| \leq 2 n A \epsilon^{*}\left|x-x^{0}\right|^{m-\sigma_{k}+1} .
$$

Given $\epsilon^{\prime}>0$, set $\epsilon^{*}=\epsilon^{\prime} /(2 n A)$, and choose $\delta$ accordingly; this inequality then completes the proof.

\section{REFERENCES}

1. A. E. Currier, Proof of the fundamental theorems on second order cross partial derivatives, Trans. Amer. Math. Soc. 35 (1933), 245-253.

2. H. Federer, Surface Area II, Trans. Amer. Math. Soc. 55 (1944), 438-456.

3. M. D. Kirszbraun, Über die zusammenziehenden und Lipschitzschen Transformationen, Fund. Math. 22 (1934), 77-108.

4. E. J. MacShane, Extension of range of functions, Bull. Amer. Math. Soc. 40 (1934), 837-842.

5. J. Marcinkiewicz, Sur les séries de Fourier, Fund. Math. 27 (1936), 38-69. 
6. S. Saks, Theory of the integral, Second Edition, Monografje Matematyczne, WarszawaLwow, 1937.

7. H. Whitney, Analytic extensions of differentiable functions defined in closed sets, Trans. Amer. Math. Soc. 36 (1934), 63-89.

8. 50 (1944), 76-81.

HARVARD UNIVERSITY 



\title{
EDITORS
}

\author{
HERBERT BUSEMANN \\ University of Southern California \\ Los Angeles 7, California
}

R. M. RoBINSON

University of California

Berkeley 4, California

E. F. BECKENBACH, Managing Editor

University of California

Los Angeles 24, California

ASSOCIATE EDITORS
R. P. DILWORTH
P. R. HALMOS
BQRGE JESSEN
J. J. STOKER
HERBERT FEDERER
HEINZ HOPF
PAUL LÉVY
E. G. STRAUS
MARSHALL HALL
R. D. JAMES
GEORGE PÓLYA
KÔSAKU YOSIDA

\section{SPONSORS}

UNIVERSITY OF BRITISH COLUMBIA

CALIFORNIA INSTITUTE OF TECHNOLOGY UNIVERSITY OF CALIFORNIA, BERKELEY

UNIVERSITY OF CALIFORNIA, DAVIS

UNIVERSITY OF CALIFORNIA, LOS ANGELES

UNIVERSITY OF CALIFORNIA, SANTA BARBARA

OREGON STATE COLLEGE

UNIVERSITY OF OREGON
UNIVERSITY OF SOUTHERN CALIFORNIA STANFORD UNIVERSITY

WASHINGTON STATE COLLEGE

UNIVERSITY OF WASHINGTON

AMERICAN MATHEMATICAL SOCIETY NATIONAL BUREAU OF STANDARDS, INSTITUTE FOR NUMERICAL ANALYSIS

\section{Vari-Type Composition by \\ Cecile Leonard \\ Ruth Stafford}

With the cooperation of

E. F. Beckenbach

E. G. Straus

Printed in the United States of America by

Edwards Brothers, Inc., Ann Arbor, Michigan

UNIVERSITY OF CALIFORNIA PRESS - BERKELEY AND LOS ANGELES COPYRIGHT 1951 BY PACIFIC JOURNAL OF MATHEMATICS 


\section{Pacific Journal of Mathematics}

\section{Vol. 1, No. $1 \quad$ November, 1951}

Ralph Palmer Agnew, Ratio tests for convergence of series............. 1

Richard Arens and James Dugundji, Topologies for function spaces....... 5

B. Arnold, Distributive lattices with a third operation defined ........... 33

R. Bing, Concerning hereditarily indecomposable continua ........... 43

David Dekker, Generalizations of hypergeodesics ............... 53

A. Dvoretzky, A. Wald and J. Wolfowitz, Relations among certain ranges of vector measures.................................... 59

Paul Erdős, F. Herzog and G. Pirani, Schlicht Taylor series whose convergence on the unit circle is uniform but not absolute .......... 75

Whilhelm Fischer, On Dedekind's function $\eta(\tau) \ldots \ldots \ldots \ldots \ldots \ldots . \ldots 3$

Werner Leutert, The heavy sphere supported by a concentrated force ...... 97

Ivan Niven and H. Zuckerman, On the definition of normal numbers ...... 103

L. Paige, Complete mappings of finite groups .................. 111

Otto Szász, On a Tauberian theorem for Abel summability ............. 117

Olga Taussky, Classes of matrices and quadratic fields ............. 127

F. Tricomi and A. Erdélyi, The asymptotic expansion of a ratio of gamma functions .................................... 133

Hassler Whitney, On totally differentiable and smooth functions ......... 143 